\title{
5-Hydroxymethylcytosine is an independent predictor of survival in malignant melanoma
}

\author{
Gerald Saldanha ${ }^{1,2}$, Kushal Joshi ${ }^{1}$, Kathryn Lawes ${ }^{2}$, Mark Bamford ${ }^{2}$, Farhaan Moosa ${ }^{1}$, \\ Kah Wee Teo $^{1}$ and J Howard Pringle ${ }^{1}$ \\ ${ }^{1}$ Department of Cancer Studies, University of Leicester, Leicester Royal Infirmary, Leicester, UK and \\ ${ }^{2}$ EMPATH Department of Cellular Pathology, University Hospitals of Leicester, Leicester Royal Infirmary, \\ Leicester, UK
}

\begin{abstract}
Outcomes for melanoma patients vary within cancer stage. Prognostic biomarkers are potential adjuncts to provide more precise prognostic information. Simple, low-cost biomarker assays, such as those based on immunohistochemistry, have strong translational potential. 5-hydroxymethylcytosine $(5 \mathrm{hmC})$ shows prognostic potential in melanoma but prior studies were small. We, therefore, analysed $5 \mathrm{hmC}$ in a retrospective cohort to provide external validation of its prognostic value. Two hundred primary melanomas were evaluated for $5 \mathrm{hmC}$ expression using immunohistochemistry. The primary objective was to assess the effect on overall survival while controlling for important confounders. Univariable and multivariable analyses were performed. REMARK guidelines were followed. The $5 \mathrm{hmC}$ immunohistochemistry scoring showed very strong inter-observer agreement (ICC 0.88) and expression was significantly related to age, site, Breslow thickness, ulceration, mitotic rate, and stage. Kaplan-Meier analysis showed $5 \mathrm{hmC}$ was associated with metastasis-free, melanomaspecific, and overall survival, $\boldsymbol{P}<\mathbf{0 . 0 0 0 1}$ for each. In univariable Cox proportional hazards models, $\mathbf{5} \mathrm{hmC}$ hazard ratios were significant and remained so in a multivariable model. A two-step cox model was created using stage and $5 \mathrm{hmC}$, as stage is the gold standard for clinical practice. The addition of $5 \mathrm{hmC}$ produced significant improvement in the model and $5 \mathrm{hmC}$ and stage were independent significant predictors. This is the largest study of the prognostic value of $5 \mathrm{hmC}$ immunohistochemistry in melanoma. The $5 \mathrm{hmC}$ scoring was easily and reproducibly performed and it was an independent predictor of metastasis-free survival, melanoma-specific survival, and overall survival. This work supports further development of $5 \mathrm{hmC}$ as a prognostic biomarker and suggests that it could add more precision to American Joint Committee on Cancer staging.

Modern Pathology (2017) 30, 60-68; doi:10.1038/modpathol.2016.159; published online 7 October 2016
\end{abstract}

Melanoma is a common and often fatal skin cancer. In clinical practice, the American Joint Committee on Cancer (AJCC) staging is the gold standard for predicting outcome at baseline. ${ }^{1,2}$ A clinical problem is that within a given AJCC stage outcome can vary, indicating a need for supplementary prognostic biomarkers to provide more precision to enable better stratification for treatment and entry into clinical trials.

Immunohistochemistry is a simple, low-cost assay that can measure biomarker expression in routinely processed tumor tissue sections. Immunohistochemistry has strong translational potential because it can be easily evaluated by a histopathologist alongside

Correspondence: Dr G Saldanha, PhD, MBChB, Department of Cancer Studies, University of Leicester, Level 3 Robert Kilpatrick Building, Leicester Royal Infirmary, Leicester LE2 7LX, UK.

E-mail: gss4@le.ac.uk

Received 27 April 2016; accepted 30 July 2016; published online 7 October 2016 traditional histological predictors. Progress in translating an immunohistochemistry biomarker into practice involves a long pathway that requires an assay that can be reproduced across different centers, a scoring system with good inter-observer agreement, a multivariable prediction model incorporating the biomarker alongside other known prognostic predictors, optimal cut-points for stratifying patients into prognostic sub-groups, and validation of the prediction model in new cases to test its ability to be generalized..$^{3,4}$ A first step in this laborious pathway is to have solid evidence that this undertaking is worthwhile, which requires a sufficiently large study that can adjust for important confounding factors. Unfortunately most immunohistochemistry biomarkers have been tested using small opportunity samples ${ }^{5-8}$ with no larger studies to provide external validation of biomarker suitability.

5-hydroxymethylcytosine $(5 \mathrm{hmC})$ is a recently described prognostic biomarker candidate that is an 
epigenetic change affecting DNA. It was only recently shown to be present in mammalian cells $s^{9,10}$ and is an oxidation product of 5methylcytosine $(5 \mathrm{mC})$ entailing conversion involving three oxidases, ten-eleven translocation (TET) 1 , TET2, and TET3. ${ }^{9-11}$ The exact biological functions of $5 \mathrm{hmC}$ remain under active investigation, but it is recognized to be important in cellular reprogramming during embryological development. ${ }^{12}$ Various human cancers have depleted levels of $5 \mathrm{hmC}$, including melanoma ${ }^{13-15}$ and mutations of genes affecting $5 \mathrm{hmC}$ levels, such as the TET family, have been found in malignant tissues. ${ }^{16,17}$ In melanoma, genome-wide mapping revealed loss of the $5 \mathrm{hmC}$ landscape in the melanoma epigenome, whereas reestablishing $5 \mathrm{hmC}$ levels suppressed melanoma growth and increased tumor-free survival in animal models. ${ }^{15}$ This same study showed that $5 \mathrm{hmC}$ levels have diagnostic value, with higher levels in benign compared with malignant melanocytic tumors. ${ }^{15}$ Other studies have subsequently supported the diagnostic value of $5 \mathrm{hmC} .{ }^{18-21}$ Preliminary evidence of prognostic value was also found, ${ }^{15}$ with lower $5 \mathrm{hmC}$ levels associated with poorer relapse-free survival, but this analysis involved only 70 melanoma patients and was limited to nodular and superficial spreading melanoma subtypes. Multivariable survival analysis of $5 \mathrm{hmC}$ levels has yet to be performed for melanoma. Other studies of $5 \mathrm{hmC}$ have also been done in melanoma, such as those assessing $5 \mathrm{hmC}$ expression during histological progression, including various forms of benign naevus, borderline tumors, and different types of melanoma, but crucially these studies did no formal survival analysis. ${ }^{18,22}$ Finally, Uchiyama et $a l^{23}$ addressed the potential value of $5 \mathrm{hmC}$ for diagnosis only and also did not assess its prognostic value.

In summary, no study has yet investigated $5 \mathrm{hmC}$ in cutaneous melanoma with both formal survival analysis and sufficient numbers to rule out confounding variables. Therefore, we undertook to fill this important gap by analyzing a large cohort of 200 cases with multivariable analysis for three clinically important prognostic end points (metastasis-free survival, melanoma-specific survival, and overall survival) to provide internal corroboration of the $5 \mathrm{hmC}$ 's prognostic value. The primary aim was to establish whether $5 \mathrm{hmC}$ is an independent predictor of overall survival while adjusting for the current prognostic gold standard, AJCC7 stage. Secondary aims were to assess metastasis-free and melanomaspecific survival and to investigate the confounding effect of other recognized prognostic factors.

\section{Materials and methods}

\section{Patients and Setting}

Cases were selected from melanoma patients diagnosed at the University Hospitals of Leicester NHS
Trust. The pathology database was searched for invasive melanomas diagnosed since 01 January 2004 and these were sequentially assessed for inclusion and exclusion criteria until a total of 200 was reached. A case was included if it was a cutaneous invasive melanoma from a patient resident in the catchment of the Leicester Hospitals and excluded if the invasive melanoma had cut out of the tissue section, if the relevant tissue blocks were too thin to cut, or if the patient had metastasis at the time of diagnosis. If the patient had multiple melanomas, the one with higher primary stage was used. This was only relevant to one patient. This individual had two primary melanomas, one AJCC stage IA from the head \& neck region and one AJCC stage IIB from the upper limb. Ipsilateral axillary node metastasis followed by subsequent distant disease and death allowed us to make a very strong inference that this was all related to the upper limb primary melanoma. We, therefore, only assessed this tumor and did not assess the head \& neck region melanoma for $5 \mathrm{hmC}$. Cases from individuals not resident in Leicester were not considered because follow-up data were not available. All the tissues were formalin-fixed and paraffin-embedded. A small biopsy was received for three melanomas, all acral, and in each a subsequent excision contained substantially more tissue and the deepest melanoma cells. We only analysed the excision and not the initial biopsy. During the time frame of this retrospective cohort, sentinel lymph node biopsy was not routinely performed and so this was not included as a variable. The study received a favorable NHS research ethics committee opinion (NRES Ethics: REC6791).

\section{Immunohistochemistry}

Immunohistochemistry was adapted from a reported method. ${ }^{15} 5$ - $\mu$ m-thick sections were prepared and heated at $65^{\circ} \mathrm{C}$ for $10 \mathrm{~min}$ then rehydrated. Sections were microwaved in citrate buffer for $20 \mathrm{~min}$ in a $750 \mathrm{~W}$ microwave. They were immersed in $2 \mathrm{~N} \mathrm{HCl}$ for $30 \mathrm{~min}$, rinsed twice in $\mathrm{H}_{2} \mathrm{O}$ for $3 \mathrm{~min}$ and treated with $100 \mathrm{mM}$ Tris+HCl pH 8.5 for $10 \mathrm{~min}$ and rinsed in water. Immunostaining was performed with the Novolink Polymer detection kit, following the manufacturer's instructions (Leica Microsystems, Milton Keynes, UK) using anti-5 hmC antibodies (clone 59.1, Active Motif, Rixensart, Belgium) diluted 1:2000 in 3\% bovine serum albumin/0.1\% Triton X 100 in TBS overnight at $4{ }^{\circ} \mathrm{C}$. The Vector SG peroxidase kit was used, producing a blue gray color (Vector Laboratories, Peterborough, UK). Sections were counterstained with filtered Nuclear Fast Red solution for $5 \mathrm{~min}$, rinsed in water, dipped in Eosin for $30 \mathrm{~s}$ and washed in water again. Sections were dehydrated and mounted with DPX. For each experiment, formalin-fixed and paraffin-embedded tonsil slides were used as positive and negative controls. Germinal centers showed weak staining, 
whereas the rest of the follicle stained strongly. Keratinocytes, inflammatory cells, and blood vessels stained strongly, acting as internal positive controls. Each batch of immunostaining included a 'no antibody' negative control.

\section{5 hmC Immunostain Scoring}

Slides were scored according to the proportion of cells with positive nuclear stain: $0(<5 \%) ; 1$ $(5-33 \%) ; 2(34-66 \%) ; 3(67-90 \%) ; 4(>90 \%)$. If more than $5 \%$ cells were positive but staining was weak throughout, the score was designated as 1 . Otherwise if more than $50 \%$ of cells showed weak staining the score was reduced by 1 . Cell morphology, rather than a double stain, was used to differentiate tumor cells from inflammatory cells, but these were readily distinguished. The assessor was blinded to the outcome at the time of scoring. The first 44 scored cases were independently scored by two raters, yielding an ICC of 0.88 , indicating very strong agreement. ${ }^{24}$

\section{Statistical Analysis}

Statistical analyses were all performed in $\mathrm{R}$ version 3.2.0. ${ }^{25}$ Survival analysis was performed using the 'survival' package. ${ }^{26} 5 \mathrm{hmC}$ was taken as an ordinal variable with five levels (0-4) for bivariable comparison with other prognostic factors using a KruskalWallis test or a $\chi^{2}$-test employing simulation with 999 replicates. Time-to-event analysis was performed with three outcomes, metastasis-free survival, melanoma-specific survival, and overall survival. For all, the date of diagnosis was taken as the date of primary sample accession in the

Table 1 Baseline melanoma data

\begin{tabular}{|c|c|c|c|}
\hline AJCC stage & & Site & \\
\hline IA & $63(31.5 \%)$ & Upper limb & $24(12 \%)$ \\
\hline IB & $71(35.5 \%)$ & Lower limb & $52(26 \%)$ \\
\hline IIA & $19(9.5 \%)$ & Trunk & $67(33.5 \%)$ \\
\hline IIB & $15(7.5 \%)$ & Head \& neck & $42(21 \%)$ \\
\hline IIC & $32(16 \%)$ & Acral & $15(7.5 \%)$ \\
\hline Age at diagnosis & & Breslow depth (mm) & \\
\hline Mean & 61 & Mean & 2.9 \\
\hline Median & 62 & Median & 1.2 \\
\hline Breslow depth (mm) & & $\begin{array}{l}\text { Mitotic rate } \\
\left(\text { per } \mathrm{mm}^{2}\right)\end{array}$ & \\
\hline Mean & 2.9 & Mean & 4.5 \\
\hline Median & 1.2 & Median & 1 \\
\hline Ulcer & & $\begin{array}{l}\text { Microscopic } \\
\text { satellites }\end{array}$ & \\
\hline Present & $46(23 \%)$ & Present & $6(3 \%)$ \\
\hline Follow-up (months) & & Gender & \\
\hline Mean & 79 & Male & $\begin{array}{c}101 \\
(50.5 \%)\end{array}$ \\
\hline Median & 83 & & \\
\hline
\end{tabular}

pathology database. For metastasis-free survival, the first metastasis (regional or distant) was considered as failure. For melanoma-specific survival, death from melanoma was considered as a failure, whereas for overall survival death from any cause was considered as failure. For metastasis-free survival, patients who remained metastasis free were censored, as were those who died without metastasis. For melanoma-specific survival, death from another cause was regarded as censoring. Survival was analysed by the Kaplan-Meier method and the log rank test. Univariable and multivariable hazard ratios were determined using the Cox proportional hazards method. Covariates were coded as follows: age, years; sex, $F=0, M=1$; site, central = head \& neck, trunk and peripheral = upper limb, lower limb, acral; Breslow thickness, millimeters; mitotic rate, mitoses per square millimeter as per hotspot method; ${ }^{27}$ ulceration, yes $=1$, no $=0$; microscopic satellites, yes $=1$, no $=0$; and AJCC7 stage, IA, IB, IIA, IIB, and IIC. Given that these seven variables are well- recognized prognostic factors, they were all entered into both univariable and multivariable analyses to ensure that $5 \mathrm{hmC}$ was not affected by weak confounders and suppressors, as recommended for sufficiently powered studies. ${ }^{28} 5 \mathrm{hmC}$ was entered as three categories: score 0, score 1-2, and score 3-4. The median follow-up was 86 months. The proportionality assumption was checked by examining plots of scaled Schoenfeld residuals against transformed time and with a goodness of fit test in the R survival package. Proportionality was not violated for the primary end points. This study adhered to REMARK guidelines, ${ }^{29}$ see Supplementary Table 1 .

\section{Results}

\section{$5 \mathrm{hmC}$ is Associated with Melanoma Prognostic Factors}

Baseline features of 200 melanomas are shown in Table 1. Bivariate contrasts were made for $5 \mathrm{hmC}$ level and established clinico-pathological prognostic features. An example of $5 \mathrm{hmC}$ staining (a score of 4 in a thin melanoma) is shown in Figure 1. A more detailed exposition of the immunostaining and scoring system is provided in the Supplementary Information. The $5 \mathrm{hmC}$ score was significantly associated with AJCC stage, age at diagnosis, site of primary tumor, Breslow depth, and ulceration. No association was found with microscopic satellites, but the number in which this feature was observed was small $(n=6)$. No association with gender was seen. Data are summarized in Table 2.

\section{5 hmC Shows Prognostic Significance}

The prognostic value of $5 \mathrm{hmC}$ was assessed using Kaplan-Meier estimates of the survivor function for 
a
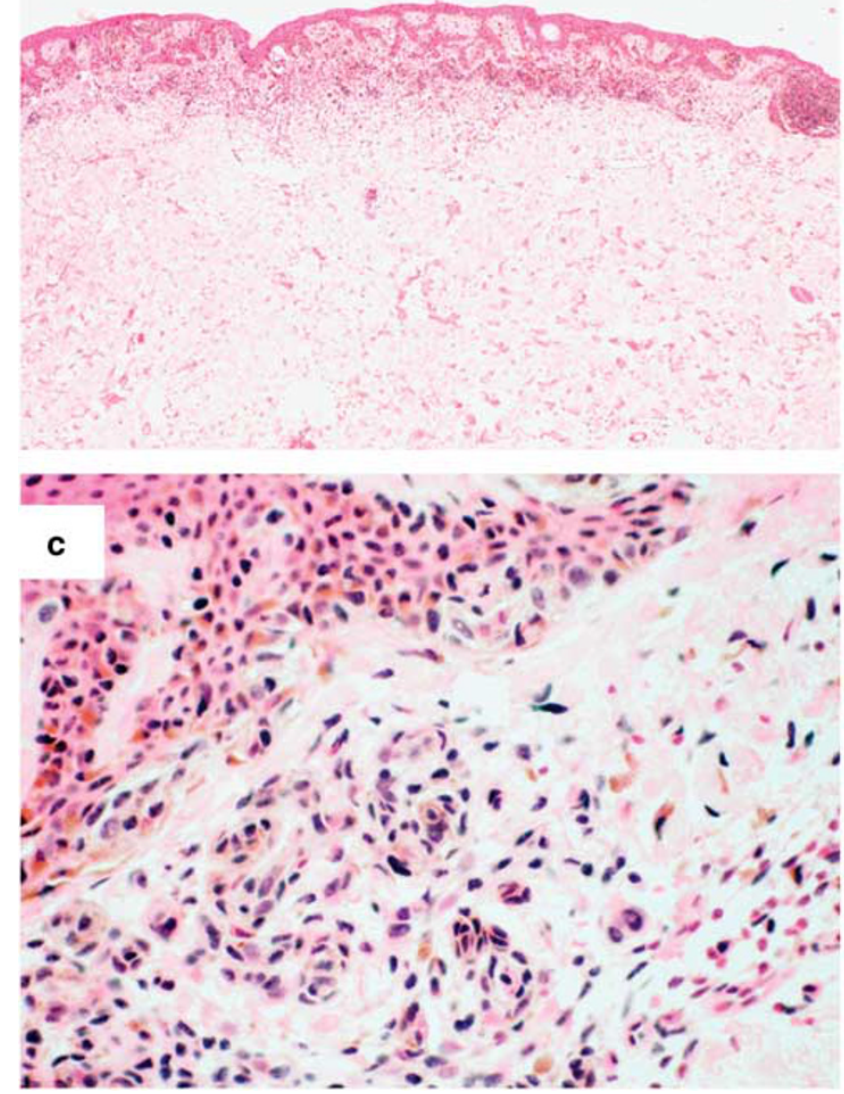

b

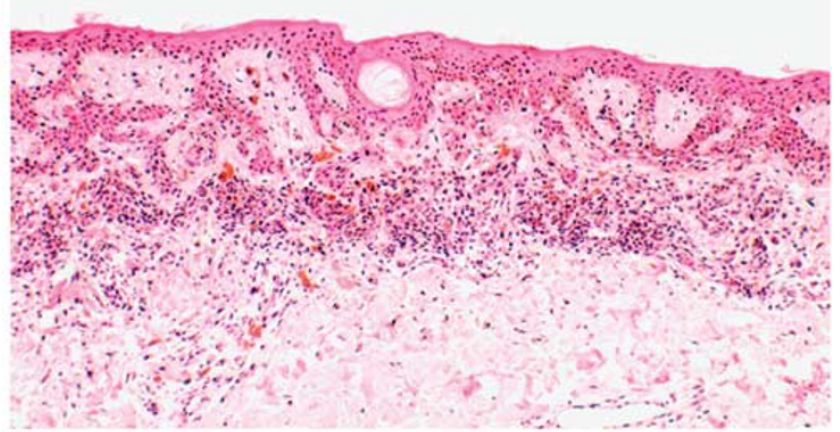

Figure 1 Thin melanoma with 5 hmC score of 4. Low-, medium-, and high-power views are shown in panels (a-c), respectively.

three types of event: metastasis-free survival, melanoma-specific survival, and overall survival. Each score from 0 to 4 was plotted as a separate curve. $5 \mathrm{hmC}$ was significantly associated with metastasis-free survival $\left(\chi^{2}=36.7\right.$ on 4 degrees of freedom, $P=2.12 \times 10^{-7}$ ), melanoma-specific survival $\left(\chi^{2}=38\right.$ on 4 degrees of freedom, $\left.P=1.14 \times 10^{-7}\right)$, and overall survival $\left(\chi^{2}=30.5\right.$ on 4 degrees of freedom, $P=3.8 \times 10^{-6}$ ), as shown in Figure 2 .

The effect of $5 \mathrm{hmC}$ on survival was next assessed with adjustment for potential confounding variables. A Cox proportional hazards regression model was fitted including $5 \mathrm{hmC}$ alongside the same established prognostic factors described in Table 2. Inspection of the Kaplan-Meier plots showed that there was an overlap of the survival curves for $5 \mathrm{hmC}$ scores 1 and 2 and for scores 3 and 4 . These groups were, therefore, combined to yield three categories for multivariable analysis: score 0 , score 1-2, and score 3-4. As this study was concerned with adjusting $5 \mathrm{hmC}$ for confounding rather than discovering an optimum prediction model, all covariates were entered simultaneously into the regression model. The results are shown in Table 3 .
Notably, 5 hmC levels were statistically significant predictors of outcome regardless of whether that was metastasis-free survival, melanoma-specific survival, or overall survival. Furthermore, significance was maintained when potential confounding covariates were added to each regression model, strongly supporting the independent prognostic value of $5 \mathrm{hmC}$.

In day-to-day clinical practice, AJCC stage is the gold standard for clinical prognostication and the primary aim of this study was to establish whether $5 \mathrm{hmC}$ could provide independent prognostic value against this gold standard. A two-step Cox proportional hazards model was developed to look at the effect of $5 \mathrm{hmC}$ after it was added to stage, focusing on overall survival, as this is the least biased outcome measure and arguably the most important. AJCC stage showed a statistically significant association with overall survival (Table 4).

Of note, $5 \mathrm{hmC}$ remained a significant predictor of overall survival when added to the proportional hazards model that included stage and overall model fit improved $\left(P=0.007, \chi^{2}=9.97, \mathrm{df}=2\right)$. However, the model's discrimination was only marginally 
Table 2 Bivariable comparisons between $5 \mathrm{hmC}$ and clinicopathological features

\begin{tabular}{|c|c|c|c|c|c|c|}
\hline & \multicolumn{5}{|c|}{5 hmC score } & \\
\hline & 0 & 1 & 2 & 3 & 4 & \\
\hline \multicolumn{7}{|l|}{ AJCC stage } \\
\hline IA & 4 & 6 & 16 & 18 & 19 & \\
\hline IB & 12 & 22 & 20 & 12 & 5 & \\
\hline IIA & 6 & 6 & 4 & 2 & 1 & \\
\hline IIB & 0 & 5 & 9 & 1 & 0 & \\
\hline IIC & 15 & 9 & 5 & 3 & 0 & $P=0.001$ \\
\hline \multicolumn{7}{|l|}{ Age } \\
\hline Mean years & 64 & 63 & 67 & 53 & 53 & $P=5.34 \times 10^{-5}$ \\
\hline \multicolumn{7}{|l|}{ Gender } \\
\hline Male & 19 & 24 & 28 & 15 & 15 & \\
\hline Female & 18 & 24 & 26 & 21 & 10 & $P=0.72$ \\
\hline \multicolumn{7}{|l|}{ Site } \\
\hline Upper limb & 8 & 8 & 3 & 2 & 3 & \\
\hline Lower limb & 10 & 10 & 17 & 15 & 0 & \\
\hline Trunk & 6 & 15 & 22 & 9 & 15 & \\
\hline Head \& neck & 8 & 11 & 8 & 9 & 6 & \\
\hline Acral & 5 & 4 & 4 & 1 & 1 & $P=0.006$ \\
\hline \multicolumn{7}{|l|}{ Breslow depth } \\
\hline Mean mm & 6.3 & 2.8 & 2.5 & 1.8 & 0.7 & $P=7.03 \times 10^{-8}$ \\
\hline \multicolumn{7}{|l|}{ Mitotic rate } \\
\hline Mean per $\mathrm{mm}^{2}$ & 8.8 & 6.0 & 4.1 & 1.6 & 0.4 & $P=3.11 \times 10^{-9}$ \\
\hline \multicolumn{7}{|l|}{ Ulcer } \\
\hline Absent & 21 & 34 & 43 & 32 & 24 & \\
\hline Present & 16 & 14 & 11 & 4 & 1 & $P=0.0014$ \\
\hline \multicolumn{7}{|l|}{ Microsatellites } \\
\hline Absent & 34 & 47 & 52 & 36 & 25 & \\
\hline Present & 3 & 1 & 2 & 0 & 0 & $P=0.26$ \\
\hline
\end{tabular}

improved by the addition of $5 \mathrm{hmC}$, as the concordance index for stage alone and for stage plus $5 \mathrm{hmC}$ changed minimally from 0.80 to 0.81 and the $R^{2}$ also changed only slightly, from 0.38 to 0.41 . This perhaps reflects the fact that no single biomarker is likely to be of value on its own, but rather would benefit from being part of a biomarker panel.

\section{Discussion}

The present study shows that $5 \mathrm{hmC}$ is associated with melanoma prognostic factors and that it is an independent prognostic factor for metastasis-free survival, melanoma-specific survival, and overall survival when adjusted for other confounding prognostic factors, including the clinical gold standard, AJCC stage.

A relatively high proportion of our cases showed strong staining, ie, scored 4 , for $5 \mathrm{hmC}$, which differs from earlier studies. These differences are likely to be the factors that will vary between studies such as differences between case mixes, types of tissue (tissue microarray or whole sections), technical differences in staining protocol, and differences in the approach to scoring. For instance, one prior study used a tissue microarray that included many metastatic cases. ${ }^{21}$ On the other hand, we used a true cohort based on date of accession into the hospital pathology laboratory and so our study is enriched for thinner melanomas falling into AJCC stages IA and IB. Because $5 \mathrm{hmC}$ is known to be reduced with progression, we speculate that this could be an important reason why many of our cases showed strong staining.

Results have some need for cautious interpretation. $5 \mathrm{hmC}$ remained significant when added to AJCC stage in the survival model but the effect on discrimination (concordance index and $R^{2}$ ) was minimal suggesting that only a small amount of variation is explained beyond that already explained by stage. It is unlikely that any individual biomarker would lead to substantially increased discrimination alone and a panel of biomarkers is the likely way forward. It is also important to note that sentinel node biopsy was not routinely performed on the cases used in this study. The therapeutic benefit of sentinel node biopsy remains unproven, but there is consensus that this procedure is a powerful prognostic factor and its use has been supported internationally by AJCC and in the UK, NICE recommends that it should be offered to appropriate individuals. If $5 \mathrm{hmC}$ is to be used in future for prognostic modeling, it is important that models are constructed in two formats: one without SLNB status for those who choose not to have the procedure, and one including SLNB status for the rest. This approach has been used previously. ${ }^{30}$ There is a debate about which covariates to include in regression models. In this study, well-recognized clinical prognostic factors (age at diagnosis, sex, and site of origin) and histological features used for staging (Breslow depth, ulceration, mitotic index, and microsatellitosis) were included. Other variables have less compelling prognostic value and were omitted. In particular, omitting some variables from regression analysis was necessary because of the constraints on model robustness if the ratio of the number of events (ie, metastasis or death) to number of covariates becomes small. ${ }^{28}$ This study is limited to patients from a single center and this has the potential to introduce bias. For example, Leicester has a relatively high proportion of Gujarati immigrants that could lead to a higher proportion of acral melanomas than other UK centers. However, we have no reason to believe that Leicester melanomas are substantially different to those elsewhere and our findings are in line with those of the smaller univariable prognostic study described by Lian et $a l^{15}$ and are consistent with studies showing reduced $5 \mathrm{hmC}$ with histological and clinical progression. ${ }^{18,22}$ It was interesting to note that Breslow thickness was not significant in multivariable analysis for any outcome. We note the very-high correlation between thickness and $5 \mathrm{hmC}$ score and 


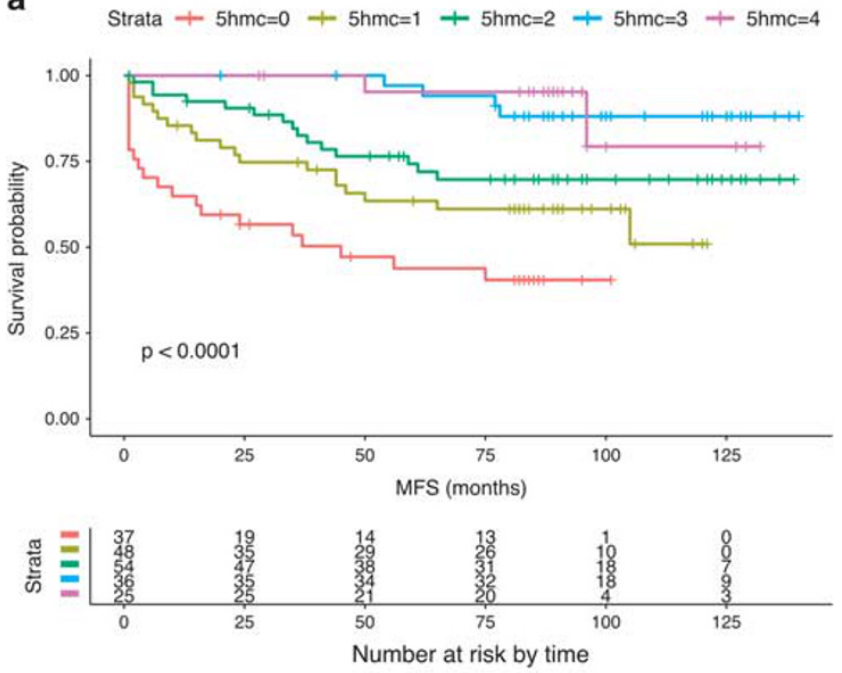

C

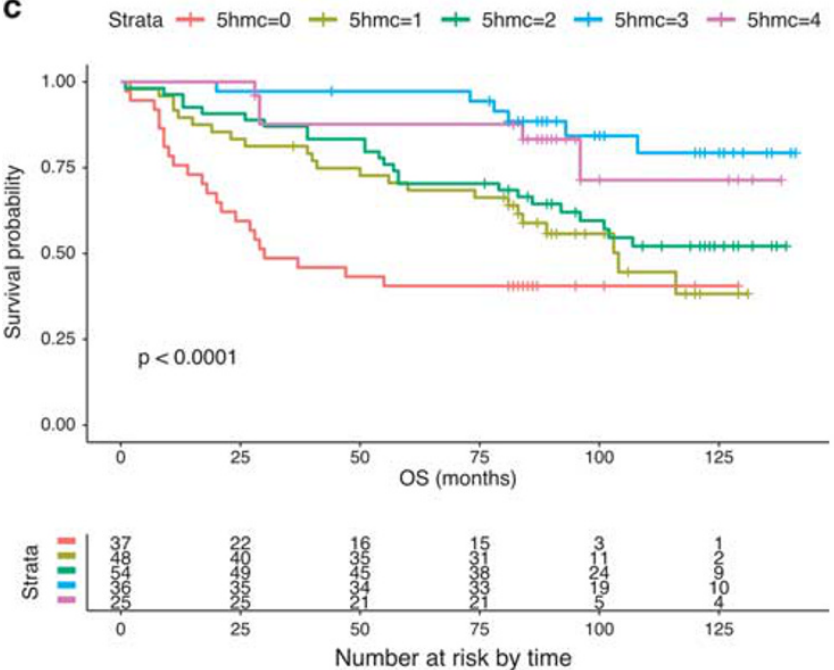

b

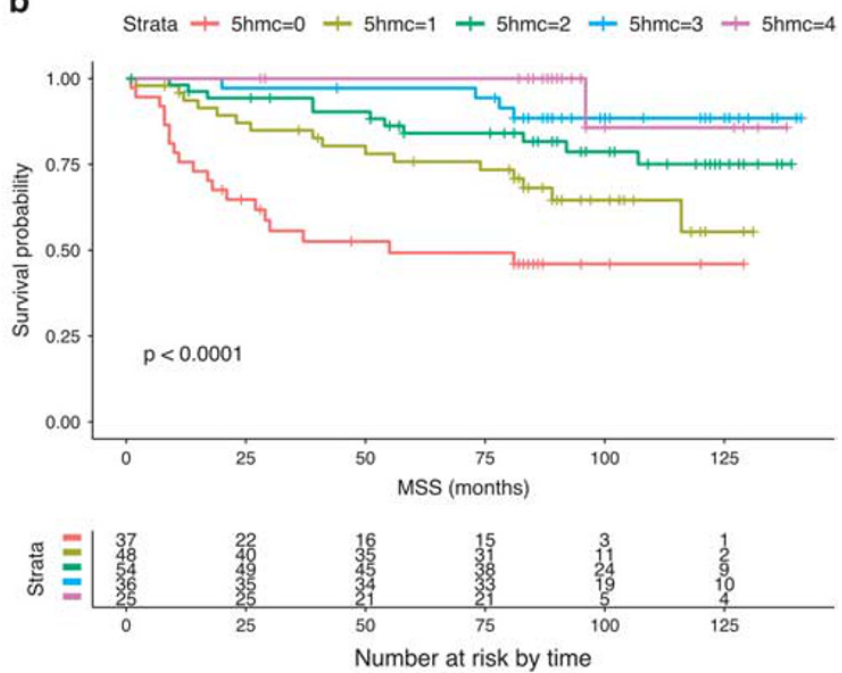

Figure 2 Kaplan-Meier plots. Plots for each 5 hmC score with different time-to-event outcomes, (a) metastasis-free, (b) melanoma-specific, and (c) overall survival.

wonder if this may have had some effect in canceling out the influence of thickness.

This study has potential clinical relevance. $5 \mathrm{hmC}$ was previously identified as a prognostic factor in melanoma ${ }^{15}$ but the number of cases was small and no analysis has to date looked at the effect of $5 \mathrm{hmC}$ on outcome after adjusting for other covariates. This study provides very strong evidence that $5 \mathrm{hmC}$ could be an independent prognostic marker for clinical use. Notably, $5 \mathrm{hmC}$ can be measured using a simple technique (immunohistochemistry) so it can be performed in any reasonably equipped clinical pathology laboratory; the antibody is monoclonal and commercially available, so it should yield reproducible results; and the scoring system is simple, has good inter-observer agreement and can be easily performed by a histopathologist using the same sections being evaluated diagnostically. All of these features increase the chance of translating this biomarker to clinical use. An image analysis-based scoring system is possible and could make scoring easier, but this is not readily available to histopathologists as they review cases at the microscope and adds complexity to biomarker translation, whereas the semi-quantitative score used in the present study has strong inter-observer agreement and is quick to perform (typically no more than $1 \mathrm{~min}$ ). The simplification of the $5 \mathrm{hmC}$ score from a 5 point score to three categories for multivariable analysis was based on the separation of the Kaplan-Meier curves, but this may have benefit moving forward because it might be argued that a 5 point score is overly precise and the simplification to three categories may be less subjective for future translation. Biomarkers for baseline prognosis are especially relevant because they can potentially stratify patients for entry into clinical trials and for sentinel node biospy. Baseline prognostic biomarkers are less relevant at present for 
Table 3 Cox proportional hazards regression for $5 \mathrm{hmC}$ and other prognostic factors

\begin{tabular}{|c|c|c|c|c|c|c|}
\hline & \multirow[b]{2}{*}{$\mathrm{n}$} & & \multicolumn{2}{|c|}{ Univariable } & \multicolumn{2}{|c|}{ Multivariable } \\
\hline & & & $H R(95 \% C I)$ & P-value & $H R(95 \% C I)$ & $\mathrm{P}$-value \\
\hline \multicolumn{7}{|l|}{$M F S^{\mathrm{a}}$} \\
\hline \multirow[t]{3}{*}{$5 \mathrm{hmC}$} & 37 & Score 0 & 1 & & 1 & \\
\hline & 102 & Score 1-2 & $0.40(0.23-0.69)$ & 0.001 & $0.50(0.27-0.93)$ & 0.02 \\
\hline & 61 & Score 3-4 & $0.10(0.04-0.24)$ & $5.0 \times 10^{-7}$ & $0.16(0.06-0.43)$ & 0.0002 \\
\hline Age & 200 & Years & $1.02(1.01-1.04)$ & 0.007 & $0.99(0.98-1.01)$ & 0.47 \\
\hline \multirow[t]{2}{*}{ Sex } & 99 & Female & 1 & & & \\
\hline & 101 & Male & $1.08(0.65-1.78)$ & 0.78 & $1.34(0.75-2.38)$ & 0.32 \\
\hline \multirow[t]{2}{*}{ Site } & 109 & Central & 1 & & 1 & \\
\hline & 91 & Peripheral & $1.30(0.79-2.15)$ & 0.30 & $1.15(0.63-2.09)$ & 0.65 \\
\hline Breslow depth & 200 & millimeters & $1.10(1.07-1.13)$ & $4.24 \times 10^{-12}$ & $1.01(0.97-1.06)$ & 0.60 \\
\hline Mitotic rate & 200 & Mitoses $/ \mathrm{mm}^{2}$ & $1.13(1.10-1.16)$ & $<2 \times 10^{-16}$ & 1.05 (1.01-1.09) & 0.007 \\
\hline \multirow{2}{*}{ Ulcer } & 154 & No & 1 & & 1 & \\
\hline & 46 & Yes & $8.64(5.13-14.53)$ & $4.44 \times 10^{-16}$ & $5.00(2.43-10.18)$ & 0.00001 \\
\hline \multirow[t]{2}{*}{ Microsatellite } & 194 & No & 1 & & 1 & \\
\hline & 6 & Yes & $3.53(1.10-11.33)$ & 0.03 & $1.18(0.35-3.98)$ & 0.79 \\
\hline \multicolumn{7}{|l|}{$M S S^{\mathrm{b}}$} \\
\hline \multirow[t]{3}{*}{$5 \mathrm{hmC}$} & 37 & Score 0 & 1 & & 1 & \\
\hline & 102 & Score 1-2 & $0.33(0.18-0.60)$ & 0.0003 & $0.43(0.21-0.86)$ & 0.02 \\
\hline & 61 & Score 3-4 & $0.09(0.03-0.24)$ & $1.67 \times 10^{-6}$ & $0.19(0.07-0.56)$ & 0.002 \\
\hline \multirow{3}{*}{$\begin{array}{l}\text { Age } \\
\text { Sex }\end{array}$} & 200 & Years & $1.03(1.01-1.05)$ & 0.006 & $1.00(0.98-1.02)$ & 0.96 \\
\hline & 99 & Female & 1 & & & \\
\hline & 101 & Male & $1.21(0.70-2.10)$ & 0.50 & $1.56(0.83-2.95)$ & 0.17 \\
\hline \multirow[t]{2}{*}{ Site } & 109 & Central & 1 & & & \\
\hline & 91 & Peripheral & $1.52(0.87-2.64)$ & 0.14 & $1.51(0.77-2.96)$ & 0.23 \\
\hline Breslow depth & 200 & millimeters & $1.12(1.09-1.15)$ & $9.44 \times 10-15$ & $1.02(0.98-1.07)$ & 0.41 \\
\hline Mitotic rate & 200 & Mitoses $/ \mathrm{mm}^{2}$ & $1.14(1.11-1.17)$ & $<2 \times 10^{-16}$ & $1.06(1.02-1.11)$ & 0.007 \\
\hline \multirow{2}{*}{ Ulcer } & 154 & No & 1 & & & \\
\hline & 46 & Yes & $10.41(5.87-18.48)$ & $1.22 \times 10^{-15}$ & $5.16(2.34-11.40)$ & 0.00005 \\
\hline \multirow[t]{2}{*}{ Microsatellite } & 194 & No & 1 & & & \\
\hline & 6 & Yes & 4.66 (1.44-15.09) & 0.01 & $1.86(0.54-6.47)$ & 0.33 \\
\hline \multicolumn{7}{|l|}{$O S^{\mathrm{c}}$} \\
\hline \multirow[t]{3}{*}{$5 \mathrm{hmC}$} & 37 & Score 0 & 1 & & 1 & \\
\hline & 102 & Score 1-2 & $0.46(0.28-0.77)$ & 0.003 & $0.55(0.31-0.98)$ & 0.04 \\
\hline & 61 & Score 3-4 & $0.16(0.08-0.32)$ & $6.02 \times 10^{-7}$ & $0.10(0.36-0.72)$ & 0.01 \\
\hline Age & 200 & Years & $1.05(1.03-1.07)$ & $7.43 \times 10^{-8}$ & $1.03(1.01-1.04)$ & 0.009 \\
\hline \multirow[t]{2}{*}{ Sex } & 99 & Female & 1 & & & \\
\hline & 101 & Male & $1.43(0.92-2.24)$ & 0.11 & $1.64(0.97-2.79)$ & 0.07 \\
\hline \multirow[t]{2}{*}{ Site } & 109 & Central & 1 & & & \\
\hline & 91 & Peripheral & $1.12(0.72-1.74)$ & 0.62 & $1.11(0.64-1.92)$ & 0.72 \\
\hline Breslow depth & 200 & millimeters & $1.12(1.09-1.14)$ & $<2 \times 10^{-16}$ & $1.03(0.99-1.07)$ & 0.19 \\
\hline Mitotic rate & 200 & Mitoses $/ \mathrm{mm}^{2}$ & $1.13(1.11-1.16)$ & $<2 \times 10^{-16}$ & $1.08(1.04-1.12)$ & 0.00001 \\
\hline \multirow[t]{2}{*}{ Ulcer } & 154 & No & 1 & & & \\
\hline & 46 & Yes & $6.65(4.26-10.4)$ & $<2 \times 10^{-16}$ & $2.15(1.17-3.97)$ & 0.01 \\
\hline \multirow[t]{2}{*}{ Microsatellite } & 194 & No & 1 & & & \\
\hline & 6 & Yes & $6.84(2.94-15.9)$ & $7.92 \times 10^{-6}$ & $2.70(1.10-6.59)$ & 0.03 \\
\hline
\end{tabular}

a Metastasis-free survival.

${ }^{b}$ Melanoma-specific survival.

${ }^{\mathrm{c}}$ Overall survival.

newer targeted and immune therapies as these are primarily used in more advanced cancer, by which time baseline prognostic information has been superseded by the prognostic impact of distant metastasis. However, clinical trials in earlier stage disease are underway and the provision of more precise baseline prognosis may eventually become important for prioritization of high-risk patients.

Future work will be needed to determine precisely how $5 \mathrm{hmC}$ should be used. In particular, to determine how it might form part of a biomarker panel, perhaps alongside clinico-pathological features. However, this would require a substantially larger study, ideally with thousands of cases culminating in a statistical prediction model that would require external validation of the prognostic scoring system on new cases. This study provides strong support for pursuing this pathway and is supported by REMARK compliance and conforms to the six criteria for biomarkers described by Gould Rothberg et al. ${ }^{31}$

In summary, this is the largest ever study to investigate the prognostic relevance of $5 \mathrm{hmC}$ in melanoma and shows that this biomarker has 
Table 4 Cox proportional hazards regression for $5 \mathrm{hmC}$ and AJCC stage

\begin{tabular}{|c|c|c|c|c|c|c|}
\hline & \multirow[b]{2}{*}{$\mathrm{n}$} & & \multicolumn{2}{|c|}{ Univariable } & \multicolumn{2}{|c|}{ Multivariable } \\
\hline & & & $H R(95 \%$ CI $)$ & P-value & $H R(95 \% C I)$ & P-value \\
\hline \multicolumn{7}{|l|}{$O S^{\mathrm{a}}$} \\
\hline \multirow{3}{*}{$5 \mathrm{hmC}$} & 37 & Score 0 & See Table 3 & See Table 3 & 1 & \\
\hline & 102 & Score $1-2$ & See Table 3 & See Table 3 & $0.52(0.30-0.89)$ & 0.02 \\
\hline & 61 & Score $3-4$ & See Table 3 & See Table 3 & $0.30(0.14-0.65)$ & 0.002 \\
\hline \multirow[t]{5}{*}{ AJCC Stage } & 63 & IA & 1 & & 1 & \\
\hline & 71 & IB & 3.03 (1.29-7.13) & 0.01 & $2.41(1.00-5.78)$ & 0.049 \\
\hline & 19 & IIA & $6.99(2.71-18.05)$ & 0.00006 & $5.04(1.90-13.35)$ & 0.001 \\
\hline & 15 & IIB & $9.37(3.56(24.66)$ & $5.9 \times 10^{-6}$ & $8.12(2.99-22.06)$ & 0.00004 \\
\hline & 32 & IIC & $28.58(12.38-66.02)$ & $4.1 \times 10^{-15}$ & $21.41(8.99-51.01)$ & $4.6 \times 10^{-12}$ \\
\hline
\end{tabular}

${ }^{\mathrm{a}}$ Overall survival.

independent prognostic value for predicting metastasis-free survival, melanoma-specific survival, and overall survival. Given that translation of a biomarker is not trivial, strong evidence is needed to prioritize a biomarker for further translation. We, here, provide that evidence for $5 \mathrm{hmC}$.

\section{Disclosure/conflict of interest}

The authors declare no conflict of interest.

\section{References}

1 Marsden JR, Newton-Bishop JA, Burrows L, et al. Revised U.K. guidelines for the management of cutaneous melanoma 2010. Br J Dermatol 2010;163: 238-256.

2 National Comprehensive Cancer Network. NCCN Guidelines version 2.2016. Melanoma. Available at: http://www.nccn.org/professionals/physician_gls/pdf/ melanoma.pdf 2016, (accessed on 10 March 2016).

3 Moore DA, Pringle JH, Saldanha GS. Prognostic tissue markers in melanoma. Histopathology 2012;60: 679-689.

4 Ransohoff DF. Rules of evidence for cancer molecularmarker discovery and validation. Nat Rev Cancer 2004;4:309-314.

5 Gould Rothberg BE, Berger AJ, Molinaro AM, et al. Melanoma prognostic model using tissue microarrays and genetic algorithms. J Clin Oncol 2009;27: 5772-5780.

6 Gould Rothberg BE, Rimm DL. Biomarkers: the useful and the not so useful-an assessment of molecular prognostic markers for cutaneous melanoma. J Invest Dermatol 2010;130:1971-1987.

7 Mandala M, Massi D. Tissue prognostic biomarkers in primary cutaneous melanoma. Virchows Arch 2014;464: 265-281.

8 Schramm SJ, Mann GJ. Melanoma prognosis: a REMARK-based systematic review and bioinformatic analysis of immunohistochemical and gene microarray studies. Mol Cancer Therap 2011;10:1520-1528.

9 Kriaucionis S, Heintz N. The nuclear DNA base 5-hydroxymethylcytosine is present in Purkinje neurons and the brain. Science 2009;324:929-930.
10 Tahiliani M, Koh KP, Shen Y, et al. Conversion of 5-methylcytosine to 5-hydroxymethylcytosine in mammalian DNA by MLL partner TET1. Science 2009;324: 930-935.

11 Ito S, D'Al4essio AC, Taranova OV, et al. Role of Tet proteins in $5 \mathrm{mC}$ to $5 \mathrm{hmC}$ conversion, ES-cell selfrenewal and inner cell mass specification. Nature 2010;466:1129-1133.

12 Pfeifer GP, Kadam S, Jin SG. 5-hydroxymethylcytosine and its potential roles in development and cancer. Epigenetics Chromatin 2013;6:10.

13 Haffner MC, Chaux A, Meeker AK, et al. Global 5hydro-

xymethylcytosine content is significantly reduced in tissue stem/progenitor cell compartments and in human cancers. Oncotarget 2011;2:627-637.

14 Jin SG, Jiang Y, Qiu RX, et al. 5Hydroxymethylcytosine is strongly depleted in human cancers but its levels do not correlate with IDH1 mutations. Cancer Res 2011;71:7360-7365.

15 Lian CG, $\mathrm{Xu} \mathrm{Y}$, Ceol C, et al. Loss of 5-hydroxymethylcytosine is an epigenetic hallmark of melanoma. Cell 2012;150:1135-1146.

16 Lee JJ, Sholl LM, Lindeman NI, et al. Targeted nextgeneration sequencing reveals high frequency of mutations in epigenetic regulators across treatment-naive patient melanomas. Clin Epigenetics 2015;7:59.

17 Song F, Amos CI, Lee JE, et al. Identification of a melanoma susceptibility locus and somatic mutation in TET2. Carcinogenesis 2014;35:2097-2101.

18 Larson AR, Dresser KA, Zhan Q, et al. Loss of 5hydroxymethylcytosine correlates with increasing morphologic dysplasia in melanocytic tumors. Mod Pathol 2014;27:936-944.

19 Lee JJ, Cook M, Mihm MC, et al. Loss of the epigenetic mark, 5-hydroxymethylcytosine, correlates with small cell/nevoid subpopulations and assists in microstaging of human melanoma. Oncotarget 2015;6:37995-38004.

20 Lee JJ, Granter SR, Laga AC, et al. 5-Hydroxymethylcytosine expression in metastatic melanoma versus nodal nevus in sentinel lymph node biopsies. Mod Pathol 2015;28:218-229.

21 Rodic N, Zampella J, Sharma R, et al. Diagnostic utility of 5-hydroxymethylcytosine immunohistochemistry in melanocytic proliferations. J Cutan Pathol 2015;42: 807-814.

22 Gambichler T, Sand M, Skrygan M. Loss of 5-hydroxymethylcytosine and ten-eleven translocation 
2 protein expression in malignant melanoma. Melanoma Res 2013;23:218-220.

23 Uchiyama R, Uhara H, Uchiyama A, et al. 5-Hydroxymethylcytosine as a useful marker to differentiate between malignant melanomas and benign melanocytic nevi. J Dermatol Sci 2014;73:161-163.

24 Landis JR, Koch GG. The measurement of observer agreement for categorical data. Biometrics 1977;33:159-174.

$25 \mathrm{R}$ Core Team. A Language and Environment for Statistical Computing. Vienna, Austria: R Foundation for Statistical Computing. 2013.

26 Therneau T. A Package for Survival Analysis in S. version 2.38http://CRAN.R-project.org/package = survival 2015.

27 Balch CM, Gershenwald JE, Soong SJ, et al. Final version of 2009 AJCC melanoma staging and classification. J Clin Oncol 2009;27:6199-6206.
28 Katz MH. Multivariable analysis: a primer for readers of medical research. Ann Intern Med 2003;138: $644-650$.

29 McShane LM, Altman DG, Sauerbrei W, et al. Reporting recommendations for tumor marker prognostic studies. J Clin Oncol 2005;23:9067-9072.

30 Maurichi A, Miceli R, Camerini T, et al. Prediction of survival in patients with thin melanoma: results from a Multi-Institution Study. J Clin Oncol 2014;32: 2479-2485.

31 Gould Rothberg BE, Bracken MB, Rimm DL. Tissue biomarkers for prognosis in cutaneous melanoma: a systematic review and meta-analysis. J Natl Cancer Inst 2009;101:452-474.

Supplementary Information accompanies the paper on Modern Pathology website (http://www.nature.com/ modpathol) 